\title{
Correlation between Abdominal Ultrasonographic Findings and CD4 Cell Count in Adult Patients with HIV/AIDS in Jos, Nigeria
}

\author{
D. Atsukwei' ${ }^{1}$ E. D. Eze ${ }^{2}$, N. D. Chom ${ }^{3}$, E. O. Igoh'1, S. C. Owoeye ${ }^{1}$, A. Angbalaga', D. A. Akut ${ }^{1}$ \\ ${ }^{1}$ Department of Radiology, Jos University Teaching Hospital, Jos, Nigeria \\ ${ }^{2}$ Department of Physiology, Faculty of Biomedical Sciences, Kampala International University, Kampala, Uganda \\ ${ }^{3}$ Department of Radiology, Ahmadu Bello University Teaching Hospital, Zaria, Nigeria \\ Email: *daniel.ejike@kiu.ac.ug
}

How to cite this paper: Atsukwei, D., Eze, E.D., Chom, N.D., Igoh, E.O., Owoeye, S.C., Angbalaga, A. and Akut, D.A. (2017) Correlation between Abdominal Ultrasonographic Findings and CD4 Cell Count in Adult Patients with HIV/AIDS in Jos, Nigeria. Advances in Molecular Imaging, 7, 49-66. https://doi.org/10.4236/ami.2017.73003

Received: July 8, 2017

Accepted: September 4, 2017

Published: September 7, 2017

Copyright ( 2017 by authors and Scientific Research Publishing Inc. This work is licensed under the Creative Commons Attribution International License (CC BY 4.0).

http://creativecommons.org/licenses/by/4.0/

\begin{abstract}
Acquired Immunodeficiency Syndrome (AIDS) is caused by Human Immunodeficiency Viruses (HIV) resulting in progressive destruction of cell mediated immunity. The abdominal manifestations of AIDS are related to the level of $\mathrm{CD}_{4}^{+}$cells count as well as viral load. Abdominal ultrasound examination is easy to perform, non-invasive, inexpensive, readily available and reproducible investigation which provides valuable information about abdominal findings in AIDS. The objective of the study was to evaluate abdominal ultrasound findings in adult HIV/AIDS patients in Jos, Plateau State, Nigeria and correlate these findings with the patients' $\mathrm{CD}_{4}^{+}$counts. A cross-sectional study of abdominal ultrasound findings of adult patients with HIV/AIDS was conducted over a period of six months. The abdominal ultrasound findings and $\mathrm{CD}_{4}^{+}$counts were studied. Two hundred (40\%) of the patients had normal abdominal ultrasound, while $60 \%$ (300) had various abnormalities. The common abnormalities included increased liver parenchymal echogenicity in $25.0 \%$, hepatomegaly in $23.4 \%$, splenomegaly in $6.6 \%$, increased splenic echogenicity in $6.2 \%$ and thickened gallbladder wall in $12.6 \%$, elevated renal parenchymal echogenicity in $6.4 \%$, enlarged kidneys in $2.6 \%$, lymphadenopathy in $6.0 \%$, and ascites in $2.4 \%$. Pelvic abscess was the least pathology in $0.2 \%$. Most of the findings did not correlate with the patients' $\mathrm{CD}_{4}^{+}$count except for lymphadenopathy and ascites. Although abdominal ultrasound examination is invaluable in the management of these patients, however, it has not shown to be useful in predicting the patients' immune status.
\end{abstract}

\section{Keywords}

Abdominal Ultrasonography, Adult Patients, CD4 Cell Count, HIV/AIDS 


\section{Introduction}

The Acquired Immunodeficiency Syndrome (AIDS) is caused by the Human Immunodeficiency Virus (HIV). The infection causes progressive destruction of the cell mediated immune (CMI) system, primarily by eliminating $\mathrm{CD}_{4}^{+} \quad \mathrm{T}$-helper lymphocytes [1]. The degree of immunodeficiency is related to the level of the $\mathrm{CD}_{4}^{+}$count and, as such, $\mathrm{CD}_{4}^{+}$count is a good index for monitoring the disease's progression. Though the major target of the virus is the immune system, the frequency of abdominal disorders in HIV/AIDS patients has been reported to be second only to pulmonary disease [2]. Radiological procedures are essential in both diagnostic and interventional roles. Computed Tomography (CT) and Magnetic Resonance Imaging (MRI) better characterize HIV-related abdominal diseases but are expensive and are scarce in the typically impoverished, HIV-infected sub-Saharan region [3]. Ultrasonography is easy to perform, non-invasive, inexpensive and safe imaging technique that is available in Africa where AIDS is most prevalent [2]. Abdominal Ultrasonography can be used to examine the abdominal organs including the liver, gallbladder, spleen, lymph nodes, pancreas and the kidneys which are commonly affected by opportunistic infections and malignancies [4].

Liver disease is an increasingly important cause of morbidity and mortality in patients with HIV/AIDS. With the availability of Highly Active Anti-Retroviral Therapy (HAART), there has been an observable change in the pattern of liver disease in these patients [5]. Although opportunistic infections and neoplasms are still seen, co-infection with hepatitis viruses, especially Hepatitis $C$ virus is now emerging as the most significant cause of liver disease in this group of patients [5]. In addition, drug-induced liver damage is becoming more prevalent due to the increased complexity and toxicity of the HAART regimens used [5]. Biliary disorders seen in AIDS patients can be classified into AIDS Cholangiopathy (AC), acalculus cholecystitis (ACC) and non-human immunodeficiency virus (HIV) associated disease such as gallstones. Gallstone disease is the most commonly observed cause of acute cholecystitis in this population [6]. While HIV related biliary disorders are not common, they are associated with significant morbidity when they occur. AC and ACC in HIV positive patients were first reported in 1983 by Guarda and colleagues and Pitlik et al., respectively [7] [8]. Since the advent of HAART, the incidence of AIDS associated biliary disorders have steeply declined [9] [10]. The two diseases can occur concurrently, with similar opportunistic infections causing each. Bile ducts in AIDS patients appear to be uniquely susceptible to opportunistic infections [11]. Ultrasonography, Endoscopic Retrograde Cholangio-Pancreatography (ERCP), Computed tomography (CT) scan and Magnetic Resonance Imaging (MRI) such as Magnetic Resonance Cholangio Pancreatography (MRCP) are useful in the investigation of diseases of the biliary tract [12] [13] [14] [15].

Pancreatic involvement in AIDS is very frequent (90\%) and is usually asymptomatic. Morphological changes showed three patterns of pancreatic alterations: 
"nutritional-like", inflammatory and both of these together [16]. The "nutritional-like" pattern (atrophy and steatosis) may be due to many factors such as nutritional characteristics induced by the HIV infection or related to the HIV virus itself.

Renal disease is a relatively common complication in patients with Human Immunodeficiency Virus (HIV) disease [17]. An important entity HIV Associated Nephropathy (HIVAN) can result from direct kidney infection with HIV or from the adverse effects of antiretroviral drugs [18] [19]. Further, patients with HIV disease are at risk for developing prerenal azotemia due to volume depletion resulting from salt wasting or vomiting. The affected kidneys are usually normal or large in size and highly echogenic on ultrasonograms and dense on CT scans.

Lymphadenopathy is very common among HIV-infected individuals and may occur at any stage of HIV infection [20]. Enlarged deep abdominal lymph nodes are frequently detected by ultrasound in patients with HIV infection. Ultrasound guided biopsy can also be performed [21].

Ultrasound can investigate most of the organs affected in AIDS and can guide biopsies, allowing the cyto-histological and microbiological investigations for a definitive diagnosis. Ultrasonography is a reproducible and safe imaging technique that can be used to examine the internal abdominal organs.

\section{Materials and Methods}

This was a hospital based cross-sectional study that spanned over the period of six months from September 2013 to March 2014. The study was carried out in the Department of Radiology, Jos University Teaching Hospital (JUTH), a tertiary health institution situated in the central part of Jos, Nigeria.

\subsection{Inclusion and Exclusion Criteria}

The study population comprised patients aged 18 years and above confirmed with HIV who were referred for abdominal ultrasound scan from Clinic II located within the Jos University Teaching Hospital complex. Clinic II is a specialized clinic exclusively for the management of HIV/AIDS patients. Pregnant HIV positive patients, HIV positive adults with confirmed co infection with $\mathrm{He}$ patitis A, B or C Viruses and HIV positive adults with co existing medical conditions such as Hypertension, Sickle cell disease and Diabetes Mellitus were excluded from the study. This is because these conditions could have similar ultrasonographic features with HIV/AIDS.

\subsection{Ethical Consideration}

Ethical clearance was obtained from the Research and Ethical Committee. Informed consent was obtained from the subjects before enrollment for the study. The subjects were informed of the safety of the procedure and could withdraw from the study at any stage without consequences. The data collected from the 
participants was recorded serially and kept with utmost confidentiality.

\subsection{Data Collection}

Patients were prepared by asking them to fast for 6 to 8 hours. This was to reduce bowel gas and make the gallbladder to be distended. Before the abdominal ultrasound scan, patients' blood pressures were measured using a standard sphygmomanometer to exclude hypertensive patients from the study. Western Blot and $\mathrm{CD}_{4}^{+}$results were obtained from patients' case file. Abdominal ultrasound scan examinations were performed using ALOKA SSD-3500 (Aloka Co. Ltd., Tokyo, Japan, 2007) ultrasound machine. A $3.5 \mathrm{MHz}$ curvilinear transducer was used in scanning the patients. The abdomen was sonographically examined for the various organs using standard ultrasound scanning procedures.

\subsection{Statistical Analysis}

The abdominal ultrasonographic findings and the data obtained from the structured questionnaire were entered into a computer and processed using SPSS for window version 20.0 (Microsoft Inc. Chicago, Illinois, USA, 2011). Mean \pm standard deviation was used to summarize variables. The variables were tested for correlation via the Chi-square test and cross tabulations. $\mathrm{P}$ value of 0.05 or less was considered statistically significant.

\section{Results}

A total of $500 \mathrm{HIV}$ positive patients, who met the inclusion criteria were studied. This comprised $20 \%$ males and $80 \%$ females with male to female ratio of $1: 4$. The ages of the patients ranged between 21 - 67 years with a mean and standard deviation of $40.30 \pm 9.32$ years. The age range for males and females were between 26 - 62 years and $21-67$ years with a mean and standard deviation of $45.72 \pm$ 8.89 years and $38.95 \pm 8.93$ years respectively. The predominant age group was 28 - 37 years $(36.6 \%)$ and followed closely by 38 - 47 years (33.4\%). (Table 1)

The mean $\mathrm{CD}_{4}^{+}$count was $520.55 \pm 282.67 / \mu \mathrm{l}$. The mean $\mathrm{CD}_{4}^{+}$count for males and females were $451.05 / \mu \mathrm{l}$ and $537.93 / \mu \mathrm{l}$ respectively. The minimum $\mathrm{CD}_{4}^{+}$value for males and females were $27 / \mu \mathrm{l}$ and $38 / \mu \mathrm{l}$ respectively while the

Table 1. Age and sex distribution of HIV/AIDS patients in Jos.

\begin{tabular}{cccc}
\hline & \multicolumn{3}{c}{ Sex } \\
\cline { 2 - 4 } Age (years) & $\begin{array}{c}\text { Male } \\
\text { Freq (\%) }\end{array}$ & $\begin{array}{c}\text { Female } \\
\text { Freq (\%) }\end{array}$ & $\begin{array}{c}\text { Total } \\
\text { Freq (\%) }\end{array}$ \\
\hline $18-27$ & $3(0.6)$ & $28(5.6)$ & $31(6.2)$ \\
$28-37$ & $18(3.6)$ & $165(33.0)$ & $183(36.6)$ \\
$38-47$ & $38(7.6)$ & $129(25.8)$ & $167(33.4)$ \\
$48-57$ & $31(6.2)$ & $67(13.4)$ & $98(19.6)$ \\
$58-67$ & $10(2.0)$ & $11(2.2)$ & $21(4.2)$ \\
Total & $100(20.0)$ & $\mathbf{4 0 0 ( 8 0 . 0 )}$ & $\mathbf{5 0 0 ( 1 0 0 . 0 )}$ \\
\hline
\end{tabular}


maximum $\mathrm{CD}_{4}^{+}$count were $1293 / \mu \mathrm{l}$ and $2690 / \mu \mathrm{l}$ for males and females respectively (Table 2).

Using the World Health Organization (WHO) classification of $\mathrm{CD}_{4}^{+}$immunological profile in adult HIV-infected patients [22], Two hundred and thirty six (47.2\%) patients had $\mathrm{CD}_{4}^{+}$count $\geq 500 / \mu$ (Not significant), 39.8\% had $\mathrm{CD}_{4}^{+}$ count between $350-499 / \mu \mathrm{l}$ (Mild), 4.8\% had $\mathrm{CD}_{4}^{+}$count between $200-349 / \mu \mathrm{l}$ (Advanced), while $8.2 \%$ patients had $\mathrm{CD}_{4}^{+}$count $<200 / \mu \mathrm{l}$ (Severe) (Table 3) Most of the patients in the "Not significant, "Mild", and "Severe" $\mathrm{CD}_{4}^{+}$classes were within the ages of 28 years to 47 years $(34.6 \%, 27.2 \%$, and $6.4 \%$ respectively) whereas most of the patients in the "Advanced" $\mathrm{CD}_{4}^{+}$class were within the ages of 48 years to 57 years (2.4\%) (Table 3 ). There was no statistically significant association between age and $\mathrm{CD}_{4}^{+}$counts in this study $(\mathrm{p}=0.091$, Table 3 ). Two hundred and two (40.4\%) females and 6.8\%) males were in the "Not significant" category while $30.4 \%$ females and $9.4 \%$ males were in the "Mild" class. There was statistically significant association between sex and patient's $\mathrm{CD}_{4}^{+}$counts with a $\mathrm{p}$ value of 0.01 (Table 4 ).

Two hundred (40.0\%) patients had normal abdominal ultrasound scan. Hepatomegaly was seen in $23.4 \%$ patients. Increased and decreased liver echogenicity was recorded in $25.0 \%$ and $0.6 \%$ respectively. Sixty three (12.6\%) and 15 (3.0\%) patients had gallbladder wall thickening and enlargement respectively. Gall stones and gallbladder sludge were seen in $2.4 \%$ and $2.0 \%$ of the patients respectively. Lymphadenopathy was noted in $6.0 \%$. Enlarged kidneys and increased renal echogenicity were recorded in $2.6 \%$ and $6.4 \%$ respectively. The

Table 2. $\mathrm{CD}_{4}^{+}$counts and sex distribution of HIV/AIDS patients.

\begin{tabular}{cccc}
\hline CD $_{4}^{+}$count & \multicolumn{3}{c}{ Sex } \\
\cline { 2 - 4 } & Male & Female & Total \\
\hline Mean \pm SD & $451.05 \pm 246.81$ & $537.93 \pm 282.67$ & $520.55 \pm 277.82$ \\
Min. & 27 & 38 & \\
Max. & 1293 & 2690 & \\
\hline
\end{tabular}

Table 3. Relationship between age and $\mathrm{CD}_{4}^{+}$count of HIV/AIDS patients.

\begin{tabular}{cccccc}
\hline & \multicolumn{5}{c}{$\mathbf{C D}_{4}^{+}$classification } \\
\cline { 2 - 6 } $\begin{array}{c}\text { Age group } \\
\text { (years) }\end{array}$ & $\begin{array}{c}\text { Not } \\
\text { significant } \\
\text { Freq (\%) }\end{array}$ & $\begin{array}{c}\text { Mild } \\
\text { Freq (\%) }\end{array}$ & $\begin{array}{c}\text { Advanced } \\
\text { Freq (\%) }\end{array}$ & $\begin{array}{c}\text { Severe } \\
\text { Freq (\%) }\end{array}$ & $\begin{array}{c}\text { Total } \\
\text { Freq (\%) }\end{array}$ \\
\hline $18-27$ & $13(2.6)$ & $14(2.8)$ & $2(0.4)$ & $2(0.4)$ & $31(6.2)$ \\
$28-37$ & $90(18.0)$ & $70(14.0)$ & $5(1.0)$ & $18(3.6)$ & $183(36.6)$ \\
$38-47$ & $83(16.6)$ & $66(13.2)$ & $4(0.4)$ & $14(2.8)$ & $167(33.4)$ \\
$48-57$ & $40(8.0)$ & $41(8.2)$ & $12(2.4)$ & $5(1.0)$ & $98(19.6)$ \\
$58-67$ & $10(2.0)$ & $8(1.6)$ & $1(0.2)$ & $2(0.4)$ & $21(4.2)$ \\
Total & $236(47.2)$ & $199(39.8)$ & $24(4.8)$ & $41(8.2)$ & $500(100.0)$ \\
\hline$\chi^{2}=18.903 ; \mathrm{df}=12 ; \mathrm{p}=0.091$. & & & &
\end{tabular}


Table 4. Sex distribution of $\mathrm{CD}_{4}^{+}$count in HIV/AIDS patients.

\begin{tabular}{cccc}
\hline & \multicolumn{3}{c}{ Sex } \\
\cline { 2 - 4 } $\mathbf{C D}_{4}^{+}$classification & $\begin{array}{c}\text { Male } \\
\text { Freq (\%) }\end{array}$ & $\begin{array}{c}\text { Female } \\
\text { Freq (\%) }\end{array}$ & $\begin{array}{c}\text { Total } \\
\text { Freq (\%) }\end{array}$ \\
\hline Not significant $(\geq 500)$ & $34(6.8)$ & $202(40.4)$ & $236(47.2)$ \\
Mild (350 - 499) & $47(9.4)$ & $152(30.4)$ & $199(39.8)$ \\
Advanced $(200-349)$ & $5(1.0)$ & $19(3.8)$ & $24(4.8)$ \\
Severe $(<200)$ & $14(2.8)$ & $27(5.4)$ & $41(8.2)$ \\
Total & $100(20.0)$ & $\mathbf{4 0 0 ( 8 0 . 0 )}$ & $\mathbf{5 0 0 ( 1 0 0 . 0 )}$ \\
\hline
\end{tabular}

$\chi^{2}=11.381 ; \mathrm{df}=3 ; \mathrm{p}=0.010$.

least finding was pelvic abscess which was seen in only $0.2 \%$. Incidental finding of uterine fibroid was noted in $1.8 \%$ of the patients. Some of the patients had more than one abdominal ultrasound findings (Table 5).

One hundred and seventy two (34.4\%) females and 5.6\% males had normal abdominal ultrsonographic findings while $18.6 \%$ females and $6.4 \%$ males had hyperechoic liver parenchyma. There was statistically significant association between sex and abdominal ultrasound findings ( $p=0.001$, Table 5).

The highest numbers of normal and abnormal abdominal ultrasound findings were seen in age groups 28 - 37 years and 38 - 47 years constituting $41.5 \%$ and $34.2 \%$ respectively. There was no significant correlation between age and abdominal ultrasound findings ( $\mathrm{p}=0.171$, Table 6$)$. Hyperechoic liver was the most common abnormal abdominal ultrasound finding in the age groups 28 - 37 years and 38 - 47 years accounting for $25.3 \%$ and $22.9 \%$ of the findings in these age groups respectively. Whereas thickened gallbladder wall was the commonest abnormal finding in the $58-67$ years age group accounting for $34.6 \%$ of the findings in this group (Table 7).

The "Not significant" ( $\geq 500 / \mu \mathrm{l}) \quad \mathrm{CD}_{4}^{+}$class had the highest proportion of patients with abnormal abdominal ultrasound findings. There was no correlation between the abdominal ultrasound findings and $\mathrm{CD}_{4}^{+}$count $(\mathrm{p}=0.394$, Table 8). Lymphadenopathy was the commonest abnormal abdominal ultrasound finding in the "Severe" $\mathrm{CD}_{4}^{+}$class accounting for $25.5 \%$ of the findings in this class (Table 9).

Hepatomegaly was seen in $23.4 \%$ but did not correlate with their $\mathrm{CD}_{4}^{+}$count $(\mathrm{p}=0.118$, Table 10, Figure 1$)$. Three hundred and seventy two $(74.4 \%)$ patients had normal liver echogenicity, whereas $25 \%$ had increased liver parenchymal echogenicity (Figure 2) and $0.6 \%$ patients had decreased liver parenchymal echogenicity. There was however no statistical association between liver echogenicity and $\mathrm{CD}_{4}^{+}$count (Table 10). Five (1.0\%) patients had focal hyperechoic liver masses which were confirmed histologically to be primary liver cell carcinoma and haemangioma in 2 and 3 cases respectively (Figure 3 and Figure 4).

Enlarged gallbladder (Figure 5) and gallbladder wall thickening were seen in 
Table 5. Relationship between abdominal ultrasound findings and sex of HIV/AIDS patients.

\begin{tabular}{|c|c|c|c|}
\hline \multirow[b]{2}{*}{ *Ultrasound findings } & \multicolumn{3}{|c|}{ Sex } \\
\hline & $\begin{array}{c}\text { Male } \\
\text { Freq (\%) }\end{array}$ & $\begin{array}{c}\text { Female } \\
\text { Freq (\%) }\end{array}$ & $\begin{array}{c}\text { Total } \\
\text { Freq (\%) }\end{array}$ \\
\hline Normal & $28(5.6)$ & $172(34.4)$ & $200(40.0)$ \\
\hline Hepatomegaly & $41(8.2)$ & $76(15.2)$ & $117(23.4)$ \\
\hline Hypoechoic liver & $2(0.4)$ & $1(0.2)$ & $3(0.6)$ \\
\hline Hyperechoic liver & $32(6.4)$ & $93(18.6)$ & $125(25.0)$ \\
\hline Liver mass & $1(0.2)$ & $4(0.8)$ & $5(1.0)$ \\
\hline Splenomegaly & $16(3.2)$ & $17(3.4)$ & $33(6.6)$ \\
\hline Hyperechoic spleen & $6(1.2)$ & $25(5.0)$ & $31(6.2)$ \\
\hline Thickened GB wall & $18(3.6)$ & $45(9.0)$ & $63(12.6)$ \\
\hline Gall stones & $3(0.6)$ & $9(1.8)$ & $12(2.4)$ \\
\hline Enlarged GB & $5(1.0)$ & $10(2.0)$ & $15(3.0)$ \\
\hline GB sludge & $3(0.6)$ & $7(1.4)$ & $10(2.0)$ \\
\hline Enlarged kidneys & $3(0.6)$ & $10(2.0)$ & $13(2.6)$ \\
\hline Increased renal echogenicity & $13(2.6)$ & $19(3.8)$ & $32(6.4)$ \\
\hline Hydronephrosis & $2(0.4)$ & $1(0.2)$ & $3(0.6)$ \\
\hline Renal cyst & $1(0.2)$ & $5(1.0)$ & $6(1.2)$ \\
\hline Lymphadenopathy & $8(1.6)$ & $22(4.4)$ & $30(6.0)$ \\
\hline Ascites & $6(1.2)$ & $6(1.2)$ & $12(2.4)$ \\
\hline Pelvic abscess & $0(0.0)$ & $1(0.2)$ & $1(0.2)$ \\
\hline Total & 188 & 523 & 711 \\
\hline
\end{tabular}

$\chi^{2}=42.611 ; \mathrm{df}=17 ; \mathrm{p}=0.001 . \mathrm{GB}=$ Gallbladder. ${ }^{\star}$ Some patients presented with more than one ultrasound finding.

Table 6. Relationship between age and abdominal ultrasound findings of HIV/AIDS patients.

\begin{tabular}{cccc}
\hline Age group (Years) & $\begin{array}{c}* \text { Ultrasound findings } \\
\text { Normal } \\
\text { Freq (\%) }\end{array}$ & $\begin{array}{c}\text { Abnormal } \\
\text { Freq (\%) }\end{array}$ & $\begin{array}{c}\text { Total } \\
\text { Freq (\%) }\end{array}$ \\
\hline $18-27$ & $15(7.5)$ & $23(4.5)$ & $38(5.3)$ \\
$28-37$ & $83(41.5)$ & $182(36.2)$ & $265(37.3)$ \\
$38-47$ & $63(31.5)$ & $175(34.2)$ & $238(33.5)$ \\
$48-57$ & $32(16.0)$ & $105(20.5)$ & $137(19.3)$ \\
$58-67$ & $7(3.5)$ & $26(5.1)$ & $33(4.6)$ \\
Total & $200(100)$ & $511(100)$ & $711(100)$ \\
\hline
\end{tabular}

$\chi^{2}=6.402 ; \mathrm{df}=4 ; \mathrm{p}=0.171 .{ }^{\star}$ Some patients presented with more than one ultrasound finding.

$3.0 \%$ and $12.6 \%$ respectively and $2.4 \%$ cases of cholelithiasis were seen (Figure 6). Gallbladder sludge was noted in $2.0 \%$. These findings did not individually correlate with the $\mathrm{CD}_{4}^{+}$counts $(\mathrm{p}=0.808,0.731,0.541,0.377$ respectively) (Table 10). 
Table 7. Age distribution of abnormal ultrasound findings.

\begin{tabular}{|c|c|c|c|c|c|c|c|c|}
\hline \multirow{2}{*}{\multicolumn{2}{|c|}{ Firu }} & & \multicolumn{5}{|c|}{ Age group (years) } & \multirow{2}{*}{ Total } \\
\hline & & & $18-27$ & $28-37$ & $38-47$ & $48-57$ & $58-67$ & \\
\hline \multirow{18}{*}{ 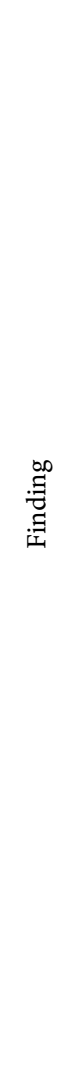 } & Hepatomegaly & Freq. (\%) & $3(13.0)$ & $45(24.7)$ & $39(22.3)$ & $25(23.8)$ & $5(19.2)$ & $117(22.9)$ \\
\hline & Hypoechoic liver & Freq. (\%) & $1(4.3)$ & $1(0.5)$ & $1(0.6)$ & $0(0.0)$ & $0(0.0)$ & $3(0.6)$ \\
\hline & Hyperechoic liver & Freq. (\%) & $5(21.7)$ & $46(25.3)$ & $40(22.9)$ & $29(27.6)$ & $5(19.2)$ & $125(24.5)$ \\
\hline & Liver mass & Freq. (\%) & $1(4.3)$ & $3(1.6)$ & $1(0.6)$ & $0(0.0)$ & $0(0.0)$ & $5(1.0)$ \\
\hline & Splenomegaly & Freq. (\%) & $1(4.3)$ & $10(5.5)$ & $12(6.9)$ & $8(7.6)$ & $2(7.7)$ & $33(6.5)$ \\
\hline & $\begin{array}{l}\text { Hyperechoic } \\
\text { spleen }\end{array}$ & Freq. (\%) & $3(13.0)$ & $13(7.1)$ & $9(5.1)$ & $4(3.8)$ & $2(7.7)$ & $31(6.1)$ \\
\hline & Thickened GB wall & Freq. (\%) & $3(13.0)$ & $17(9.3)$ & $24(13.7)$ & $10(9.5)$ & $9(34.6)$ & $63(12.3)$ \\
\hline & Gall stones & Freq. (\%) & $0(0.0)$ & $4(2.2)$ & $7(4.0)$ & $0(0.0)$ & $1(3.8)$ & $12(2.3)$ \\
\hline & Enlarged GB & Freq. (\%) & $0(0.0)$ & $7(3.8)$ & $4(2.3)$ & $4(3.8)$ & $0(0.0)$ & $15(2.9)$ \\
\hline & GB sludge & Freq. (\%) & $0(0.0)$ & $6(3.3)$ & $3(1.7)$ & $1(1.0)$ & $0(0.0)$ & $10(2.0)$ \\
\hline & Enlarged kidneys & Freq. (\%) & $1(4.3)$ & $4(2.2)$ & $6(3.4)$ & $2(1.9)$ & $0(0.0)$ & $13(2.5)$ \\
\hline & $\begin{array}{l}\text { Increased renal } \\
\text { echotexture }\end{array}$ & Freq. (\%) & $1(4.3)$ & $9(4.9)$ & $16(9.1)$ & $6(5.7)$ & $0(0.0)$ & $32(6.3)$ \\
\hline & Hydronephrosis & Freq. (\%) & $1(4.3)$ & $1(0.5)$ & $0(0.0)$ & $1(1.0)$ & $0(0.0)$ & $3(0.6)$ \\
\hline & Renal cyst & Freq. (\%) & $0(0.0)$ & $0(0.0)$ & $0(0.0)$ & $6(5.7)$ & $0(0.0)$ & $6(1.2)$ \\
\hline & Lymphadenopathy & Freq. (\%) & $3(13.0)$ & $9(4.9)$ & $9(5.1)$ & $8(7.6)$ & $1(3.8)$ & $30(5.9)$ \\
\hline & Ascites & Freq. (\%) & $0(0.0)$ & $6(3.3)$ & $4(2.3)$ & $1(1.0)$ & $1(3.8)$ & $12(2.3)$ \\
\hline & Pelvic abscess & Freq. (\%) & $0(0.0)$ & $1(0.5)$ & $0(0.0)$ & $0(0.0)$ & $0(0.0)$ & $1(0.2)$ \\
\hline & Total & Freq. (\%) & $\begin{array}{c}23 \\
(100.0)\end{array}$ & $\begin{array}{c}182 \\
(100.0)\end{array}$ & $\begin{array}{c}175 \\
(100.0)\end{array}$ & $\begin{array}{c}105 \\
(100.0)\end{array}$ & $\begin{array}{c}26 \\
(100.0)\end{array}$ & $\begin{array}{c}511 \\
(100.0)\end{array}$ \\
\hline
\end{tabular}

GB $=$ Gallbladder. ${ }^{\star}$ Some patients presented with more than one ultrasound findings.

Table 8. Abdominal ultrasound findings versus $\mathrm{CD}_{4}^{+}$count in HIV/AIDS patients.

\begin{tabular}{cccc}
\hline $\mathbf{C D}_{4}^{+}$classification & $\begin{array}{c}\text { Ultrasound findings } \\
\text { Normal } \\
\text { Freq (\%) }\end{array}$ & $\begin{array}{c}\text { Abnormal } \\
\text { Freq (\%) }\end{array}$ & $\begin{array}{c}\text { Total } \\
\text { Freq (\%) }\end{array}$ \\
\hline Not significant $(\geq 500)$ & $103(20.6)$ & $133(26.6)$ & $236(47.2)$ \\
Mild $(350-499)$ & $75(15.0)$ & $124(24.8)$ & $199(39.8)$ \\
Advanced $(200-349)$ & $9(1.8)$ & $15(3.0)$ & $24(4.8)$ \\
Severe $(<200)$ & $13(2.6)$ & $28(5.6)$ & $41(8.2)$ \\
Total & $\mathbf{2 0 0 ( 4 0 . 0 )}$ & $\mathbf{3 0 0 ( 6 0 . 0 )}$ & $\mathbf{5 0 0 ( 1 0 0 . 0 )}$ \\
\hline
\end{tabular}

$\chi^{2}=2.986 ; \mathrm{df}=3 ; \mathrm{p}=0.394$

Splenomegaly (Figure 7) and increased splenic echogenicity were seen in $6.6 \%$ and $6.2 \%$ patients respectively with no significant correlation with their $\mathrm{CD}_{4}^{+}$count $(\mathrm{p}=0.491$, Table 10$)$. Abdominal lymph nodes enlargement were noted in $6.0 \%$ which correlated significantly with their $\mathrm{CD}_{4}^{+}$counts $(\mathrm{p}=0.001$, Table 10, Figure 8). Seventy percent of the lymph nodes were seen at the porta hepatis. Some of the patients had lymphadenopathy involving multiple lymph 
Table 9. Distribution of abnormal abdominal ultrasound findings in the "Severe" $\mathrm{CD}_{4}^{+}$ class.

\begin{tabular}{ccc}
\hline *Ultrasound findings & Frequency & Percentage (\%) \\
\hline Hepatomegaly & 4 & 7.8 \\
Hyperechoic liver & 10 & 19.6 \\
Splenomegaly & 2 & 3.9 \\
Hyperechoic spleen & 5 & 9.8 \\
Thickened GB wall & 4 & 7.8 \\
Gall stones & 1 & 2.0 \\
Enlarged GB & 1 & 2.0 \\
Enlarged kidneys & 3 & 5.9 \\
Increased renal echotexture & 6 & 11.8 \\
Lymphadenopathy & 13 & 25.5 \\
Ascites & 2 & 3.9 \\
Total & 51 & 100 \\
\hline
\end{tabular}

$\mathrm{GB}=$ Gallbladder. ${ }^{\star}$ Some patients presented with more than one ultrasound finding.

Table 10. Abnormal ultrasound findings versus $\mathrm{CD}_{4}^{+}$classification.

\begin{tabular}{|c|c|c|c|c|c|c|c|}
\hline \multirow[t]{2}{*}{$\begin{array}{l}\text { Ultrasound } \\
\text { Findings }\end{array}$} & \multirow[t]{2}{*}{$\begin{array}{c}\text { Frequency } \\
\%\end{array}$} & \multicolumn{3}{|c|}{$\mathrm{CD}_{4}^{+}$classification Freq (\%) } & \multicolumn{2}{|c|}{ Chi-square } & \multirow[t]{2}{*}{$\mathrm{p}$ value } \\
\hline & & $\begin{array}{c}\text { Not } \\
\text { Significant }\end{array}$ & Mild & Advanced & Severe & & \\
\hline Hepatomegaly & $117(23.0)$ & $62(53.0)$ & $42(35.9)$ & $8(6.8)$ & $5(4.3)$ & 5.863 & 0.118 \\
\hline Hypoechoic liver & $3(0.6)$ & $2(1.1)$ & $1(0.6)$ & $0(0.0)$ & $0(0.0)$ & 0.669 & 0.880 \\
\hline Hyperechoic liver & $125(25.0)$ & $61(26.1)$ & $44(22.2)$ & $8(33.3)$ & $12(29.3)$ & 2.230 & 0.526 \\
\hline Liver mass & $5(1.0)$ & $3(1.3)$ & $2(1.0)$ & $0(0.0)$ & $0(0.0)$ & 0.832 & 0.842 \\
\hline Splenomegaly & $33(6.6)$ & $12(36.4)$ & $17(51.5)$ & $2(6.1)$ & $2(6.1)$ & 2.412 & 0.491 \\
\hline Hyperechoic spleen & $31(6.2)$ & $10(32.3)$ & $14(45.2)$ & $2(6.5)$ & $5(16.1)$ & 4.524 & 0.210 \\
\hline Thickened GB wall & $63(12.6)$ & $27(42.9)$ & $29(46.0)$ & $3(4.8)$ & $4(6.3)$ & 1.293 & 0.731 \\
\hline Gall stones & $12(2.4)$ & $4(33.3)$ & $7(58.3)$ & $0(0.0)$ & $1(8.3)$ & 2.152 & 0.541 \\
\hline Enlarged GB & $15(3.0)$ & $7(46.7)$ & $7(46.7)$ & $0(0.0)$ & $1(6.7)$ & 0.971 & 0.808 \\
\hline GB Sludge & $10(2.0)$ & $3(30.0)$ & $6(60.0)$ & $1(10.0)$ & $0(0.0)$ & 3.097 & 0.377 \\
\hline Enlarged Kidneys & $13(2.6)$ & $4(1.7)$ & $5(2.5)$ & $1(4.2)$ & $3(7.3)$ & 4.604 & 0.203 \\
\hline Increased renal echo & $32(6.4)$ & $14(43.8)$ & $12(37.5)$ & $1(3.1)$ & $5(15.6)$ & 2.630 & 0.452 \\
\hline Hydronephrosis & $3(0.6)$ & $0(0.0)$ & $3(1.5)$ & $0(0.0)$ & $0(0.0)$ & 4.565 & 0.207 \\
\hline Renal cyst & $6(1.2)$ & $1(0.4)$ & $4(2.0)$ & $1(4.2)$ & $0(0.0)$ & 4.580 & 0.205 \\
\hline Lymphadenopathy & $30(6.0)$ & $2(6.7)$ & $15(50.0)$ & $1(3.3)$ & $12(40.0)$ & 51.444 & 0.001 \\
\hline Ascites & $12(2.4)$ & $2(16.7)$ & $5(41.7)$ & $2(16.7)$ & $3(25.0)$ & 10.278 & 0.016 \\
\hline Pelvic abscess & $1(0.2)$ & $0(0.0)$ & $0(0.0)$ & $1(0.5$ & $0(0.0)$ & 1.516 & 0.679 \\
\hline Uterine fibroid & $9(1.8)$ & $4(0.8)$ & $0(0.0)$ & $3(0.6)$ & $3(0.6)$ & 0.667 & 0.717 \\
\hline
\end{tabular}




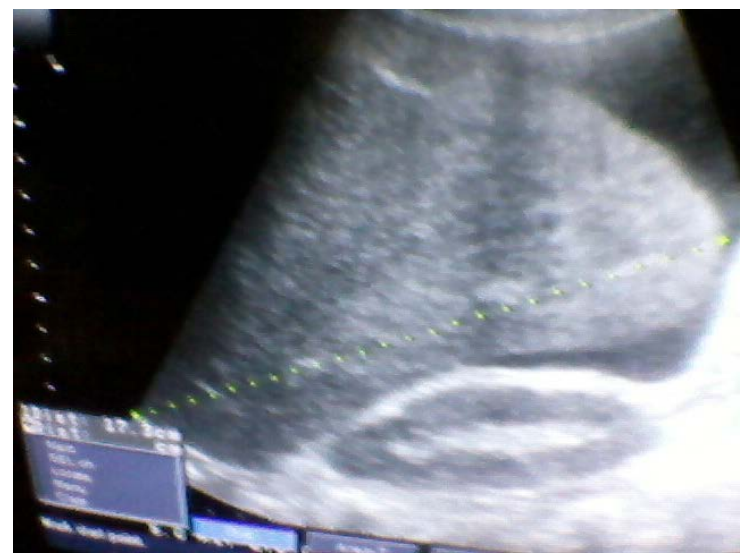

Figure 1. Longitudinal ultrasound image of the liver of a 35 year old HIV positive male showing an enlarged liver, with a cranio-caudal span of $17.3 \mathrm{~cm}$. Ascites was also noted.

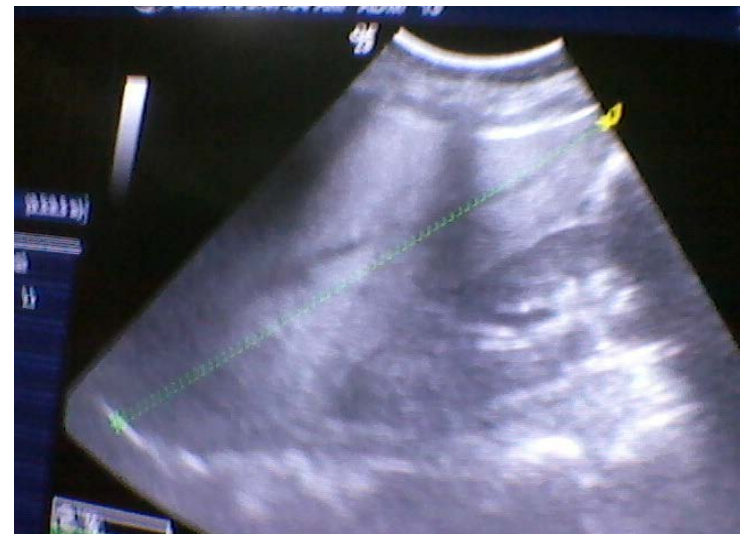

Figure 2. Longitudinal section of the liver showing increased parenchymal echogenicity in a 63 year old female HIV most likely due to steatosis (fatty infiltration) (white arrow).

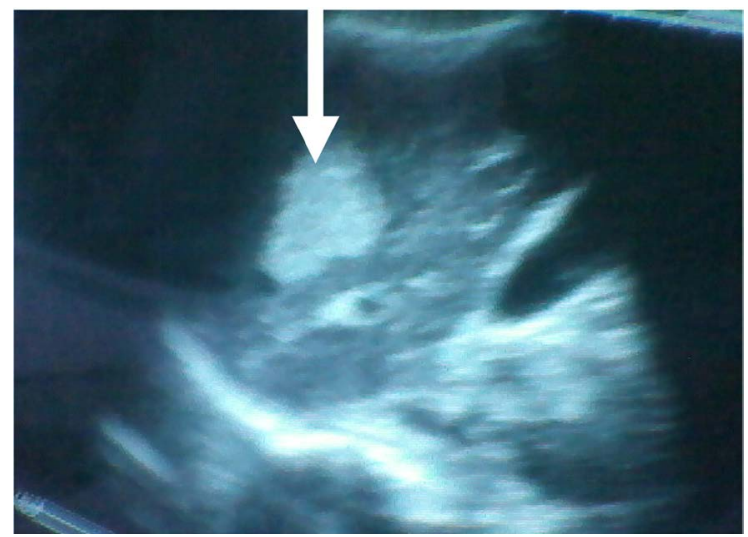

Figure 3. Longitudinal ultrasound image of the liver of a 38 year old HIV positive female showing a focal echogenic mass in the right lobe (white arrow), which turned out to be a haemangioma following biopsy. 


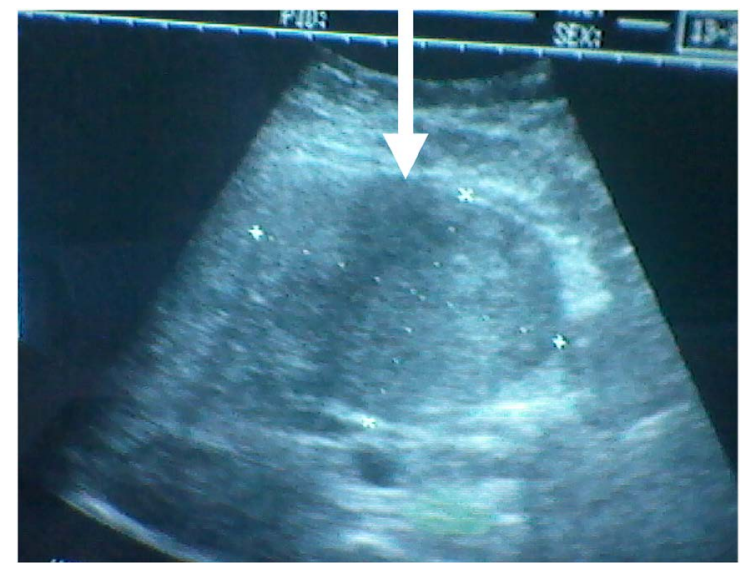

Figure 4. Transverse ultrasound image of the liver of a 53 year HIV positive male showing a focal hypoechoic mass in the left lobe (white arrow). This was histologically proven to be primary liver cell carcinoma.

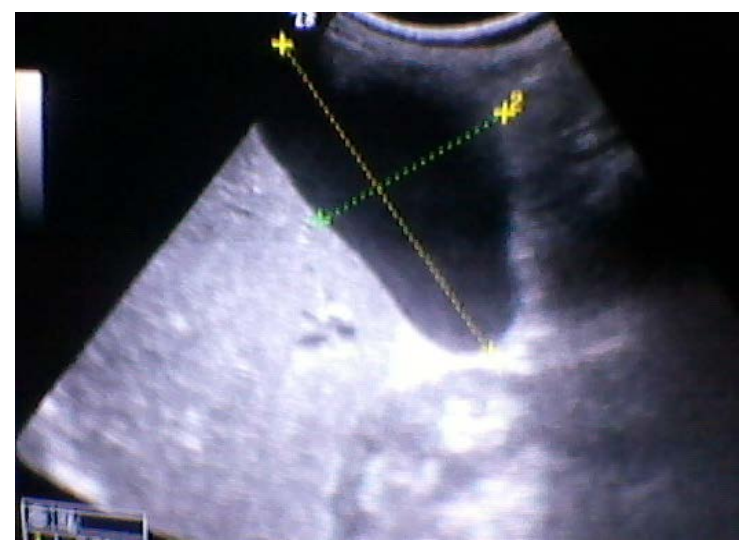

Figure 5. Longitudinal ultrasound image of the gallbladder demonstrating an enlarged gallbladder measuring $11.3 \mathrm{~cm}$ by $5.4 \mathrm{~cm}$ in a 43 year old female HIV positive patient.

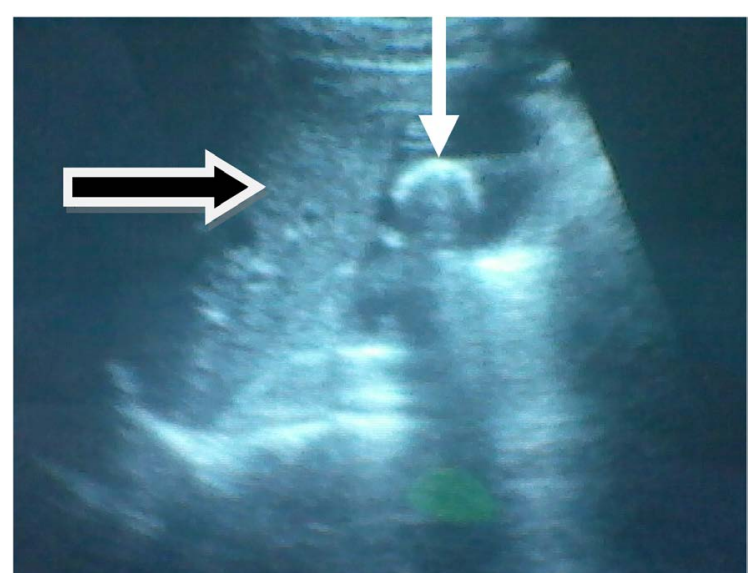

Figure 6. Longitudinal ultrasound image of the gallbladder showing a large gallstone (white arrow) in a 39 year old HIV female positive (black arrow = Liver). 


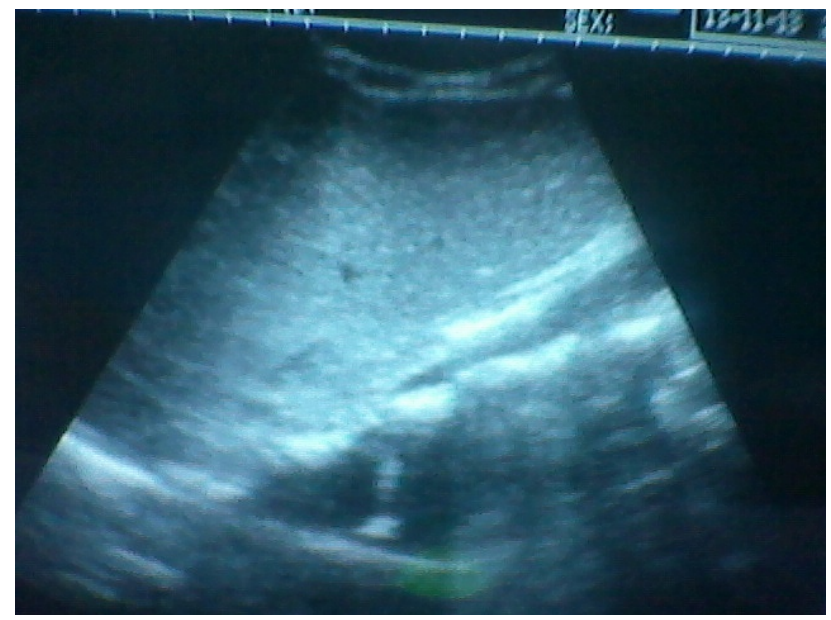

Figure 7. Longitudinal ultrasound image of the spleen in a 27 year old male HIV positive patient showing an enlarged spleen with a measured dimension of $18.5 \mathrm{~cm}$.

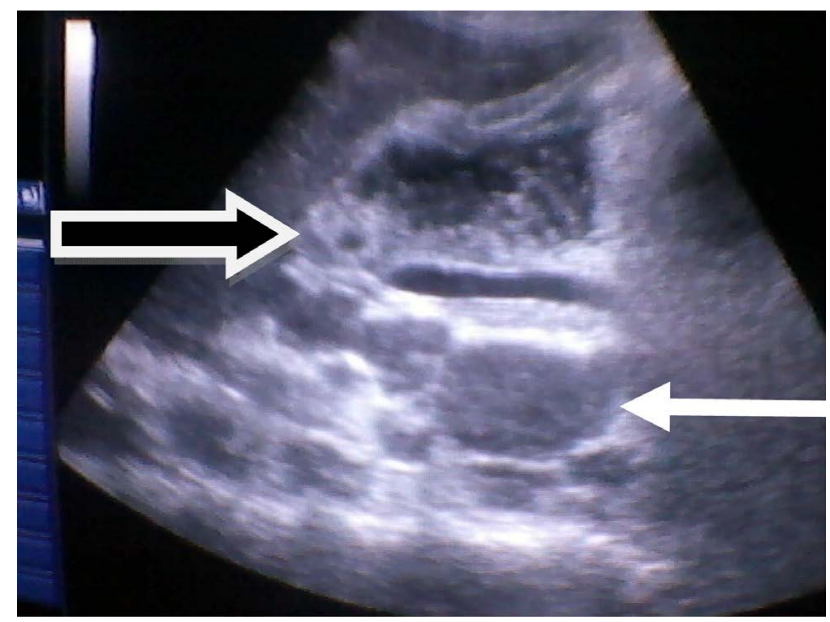

Figure 8. Transverse ultrasound of the pancreatic bed of a 36 year old HIV male patient demonstrating multiple enlarged lymph nodes (white arrow pointing to the largest lymph node) (black arrow $=$ pancreatic head).

node groups. Forty percent of those who had lympadenopathy were in the severe $\mathrm{CD}_{4}^{+}$class (Table 10).

Increased renal parenchymal echogenicity (Figure 9) and enlarged kidneys were seen in $6.4 \%$ and $2.6 \%$ ) respectively and did not correlate with their $\mathrm{CD}_{4}^{+}$ counts ( $\mathrm{p}=0.452$ and 0.203 respectively) (Table 10 ), while $93.6 \%$ had normal renal parenchymal echogenicity. Simple cortical renal cysts and calyceal dilatation were noted in $1.2 \%$ and $0.6 \%$ respectively. These findings did not correlate with the patients $\mathrm{CD}_{4}^{+}$counts (Table 10). Twelve (2.4\%) cases of ascites were seen in this study and this correlated significantly with patients $\mathrm{CD}_{4}^{+}$counts $(\mathrm{p}=$ 0.016 , Table 10). Other findings in this study included a single case of pelvic abscess (Figure 10), 5 cases of left sided pleural effusion, and 9 cases of uterine fibroids. 


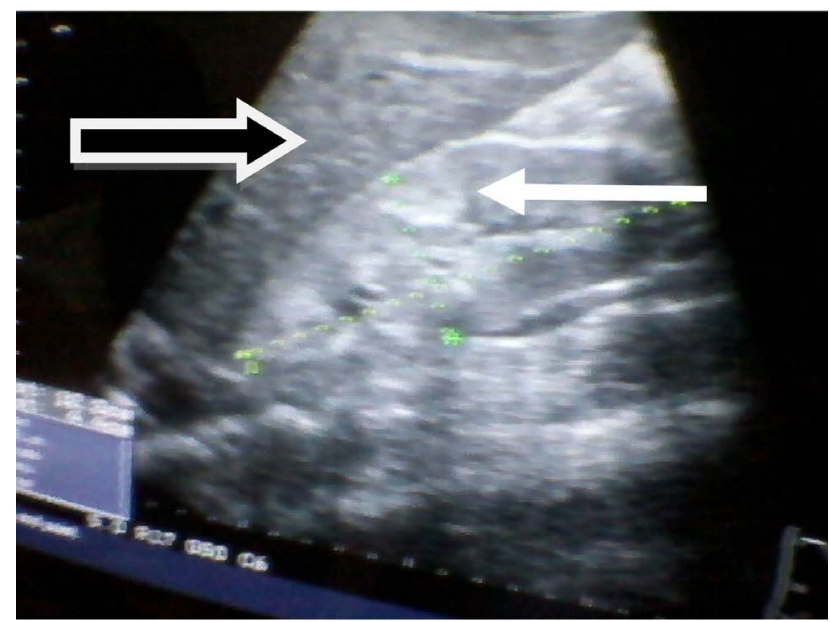

Figure 9. Longitudinal ultrasound image of the right kidney, showing increased renal cortical echogenicity (white arrow) in a 46 year old male HIV positive patient (black arrow = liver).

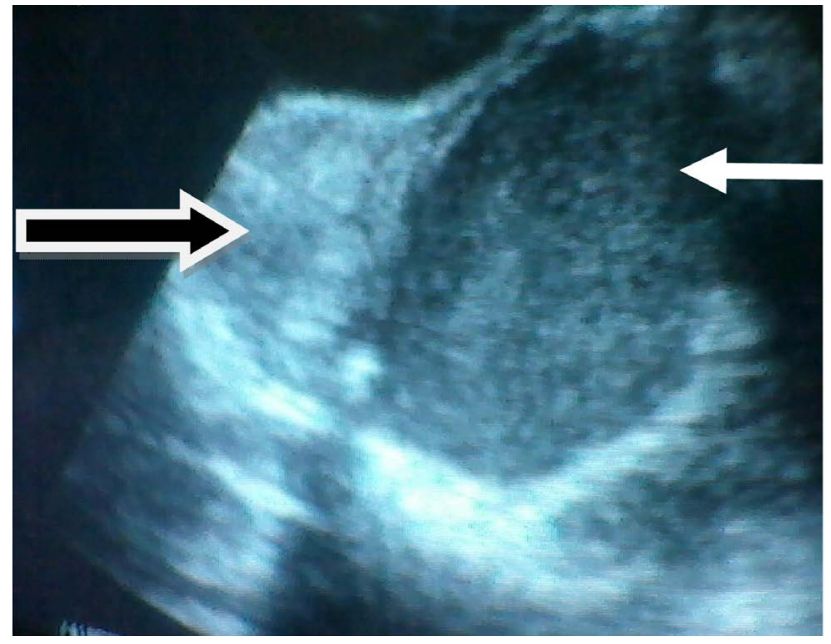

Figure 10. Transverse pelvic ultrasound image showing left sided pelvic abscess (white arrow) in a 31 year old HIV positive female patient (black arrow $=$ Uterus).

\section{Discussion}

The number of people living with HIV/AIDS worldwide is estimated to be almost 34 million [23]. The use of highly active antiretroviral therapy (HAART) has modified the disease pattern. Clinical and ultrasound findings in HIV/AIDS depend on the immune status of the patient as represented by their $\mathrm{CD}_{4}^{+}$count. The frequency of abdominal disorders in HIV/AIDS patients has been reported to be second to pulmonary disease [2]. In the majority of the sub-Saharan African countries, the absence of computed tomography facilities makes abdominal ultrasound an attractive alternative diagnostic tool in the clinical investigation of infectious and non infectious complications of HIV infected individuals [24].

The objectives of this study were to determine the frequency of occurrences of abdominal ultrasonographic findings in adults with HIV/AIDS in Jos and to 
correlate these findings with the patient's $\mathrm{CD}_{4}^{+}$counts.

A total of 500 adults with HIV/AIDS were studied which revealed majority of the patients to be females with male to female ratio of 1:4. Approximately $76 \%$ of the patients were within the age range of 18 to 47 years which corresponds with the age group at risk of HIV/AIDS and the economically productive segment of the society [25]. Igbinedion et al. in Benin-Nigeria documented about $89 \%$ of the patients to be between 18 to 47 years and a male to female ratio of 1:2.5 [3] [25]. The mean age for females and males were $38.95 \pm 8.93$ years and $45.72 \pm 8.89$ years respectively. A study done in South Western Nigeria by Obajimi et al. also documented a female preponderance $(66.5 \%)$ and female mean age of 38.02 years [2]. This was however at variance with a study in Central Africa by Tshibwabwa et al. which showed a higher male preponderance [24]. The finding of female preponderance in this study could be due to the fact that females are more prone to the disease, access health care more and are counseled during antenatal care for HIV testing leading to the discovery of their HIV status [26].

Based on the World Health Organization (WHO) classification of $\mathrm{CD}_{4}^{+}$immunological profile in adult HIV infected patients [22], 87.0\% of the patients had $\mathrm{CD}_{4}^{+}$count of $350 / \mu \mathrm{l}$ and above making up $47.2 \%$ and $39.8 \%$ for the "Not significant" and "Mild" category respectively while only $8.2 \%$ were in the "Severe" category (i.e., $\mathrm{CD}_{4}^{+}$count $<200 / \mu \mathrm{l}$ ). This was at variance with the findings of Igbinedion et al. who found majority (46.3\%) of the patients to be in the "Severe" $\mathrm{CD}_{4}^{+}$class. The high $\mathrm{CD}_{4}^{+}$count in this study may be due to early diagnosis and follow up and availability of anti retroviral drugs unlike in the past when most patients present late and were unable to access or procure anti retroviral medications. This might indicate the improved effectiveness of government interventions such as counseling and availability of drugs.

Abdominal ultrasound for diagnostic purposes in HIV/AIDS patients is requested by clinicians for a range of clinical indications such as abdominal pain and swelling, fever, hepatosplenomegaly, lymphadenopathy, abnormal liver and renal function tests. The intra abdominal organs of HIV/AIDS patients can be affected by the virus, opportunistic infections or drugs. The changes in this organs could be specific or non specific and single or multiple [2] [3] [24] [27]. This was consistent with the findings in this study (Table 5). Different abnormal abdominal sonographic findings were recorded in $60.0 \%$ (Table 8). Smith et al. [27] and Igbinedion et al. [3] recorded similar but higher abnormal abdominal sonographic findings of $81.0 \%$ and $85.0 \%$ respectively. The various abnormal abdominal sonographic findings recorded in this study were comparable with those recorded by other authors [2] [3] [24] [27]. Some of these findings were not specific to a particular pathogen or disease entity; hence, these findings did not correlate with $\mathrm{CD}_{4}^{+}$count (Table 8).

The "Not significant" $\mathrm{CD}_{4}^{+}$class had the highest number of patients with abnormal abdominal ultrasound findings (Table 8). This is at variance with the finding of Igbinedion et al. [3] who studied three hundred HIV positive patients 
in Benin-Nigeria in 2007 and recorded the highest proportion of patients with abnormal sonographic abdominal findings in the severe $\mathrm{CD}_{4}^{+}$class. This is most likely due to early diagnosis and availability of anti retroviral drugs unlike in the past when most patients present late and had limited access to anti retroviral drugs.

Hepatomegaly was the second most common abnormal abdominal sonographic findings in this study as seen in $23.4 \%$ patients. Different studies have documented figures such as $13.3 \%, 39.0 \%, 35.0 \%$ and $41.0 \%$ respectively [2] [3] [24] [27]. Hepatomegaly in these patients could be due to infections, non specific response to infective hepatitis, fatty infiltration or neoplastic infiltration from lymphoma or Kaposi sarcoma [3] [28]. Increased liver echogenicity was the most common finding as seen in $25.0 \%$. This is consistent with the findings of $18.0 \%$ and $16.5 \%$ by Igbinedion et al. [3] and Nzi et al. [29]. These findings (hepatomegaly and increased liver echotexture) did not correlate with the patients $\mathrm{CD}_{4}^{+}$ counts $(\mathrm{p}=0.118$ and 0.526 respectively, Table 10$)$. Decrease liver echogenicity was seen in only $0.6 \%$.

Focal echogenic liver masses were noted in $1.0 \%$ which did not correlate with $\mathrm{CD}_{4}^{+}$count $(\mathrm{p}=0.842$, Table 10$)$. These were confirmed histologically to be primary liver cell carcinoma and haemangioma in 2 and 3 patients respectively (Figure 4 and Figure 5). In a similar study, biopsy proven cases with similar appearance hepatic lesions to those seen in this study were described as caused by either granuloma or haemangioma [28].

Gallbladder enlargement was noted in $3.0 \%$, thickened gallbladder wall in $12.6 \%$, gall stones in $2.4 \%$ and gallbladder sludge in $2.0 \%$ patients. These findings did not correlate with $\mathrm{CD}_{4}^{+}$count $(\mathrm{p}=0.808,0.731,0.541,0.377$ respectively, Table 10). In a related study, Igbinedion et al. [3] documented thickened gallbladder wall and gall stones in $7.3 \%$ and $4.7 \%$ of the patients respectively, whereas Obajimi et al. [2] recorded gall stones in $1.3 \%$ of patients. Pawar et al. [28] in a similar study recorded thickened gallbladder wall in $9.0 \%$, gallbladder sludge in $6.0 \%$ and gall stones in $4.0 \%$ of patients which did not correlate with $\mathrm{CD}_{4}^{+}$count. None of the patients in this study showed classical features of AIDS cholangiopathy which is a sign of advanced stage of the disease [12]. This may be attributed to the fact that there is better awareness on HIV/AIDS. Therefore, most patients present early coupled with the availability of HAART now.

Splenomegaly was seen in $6.6 \%$ which did not correlate with $\mathrm{CD}_{4}^{+}$count in this study $(\mathrm{p}=0.491)$. Thirty one $(93.9 \%)$ of these patients had increased splenic echogenicity. This is consistent with the study by Obajimi et al. and Sawhey who found splenomegaly in $13.05 \%$ and $9.47 \%$ of patients respectively [2] [30]. Some studies have reported higher frequencies of splenomegaly in this group of patients [3] [28]. Splenomegaly without focal masses is common in the tropics and causes include malaria, septicaemia, typhoid, schistosomiasis, portal hypertension, haemolytic anaemia and tropical splenomegaly [3].

Thirty (6.0\%) patients had abdominal lymph node enlargement which signifi- 
cantly correlated with $\mathrm{CD}_{4}^{+}$count $(\mathrm{p}=0.001$, Table 10$)$. This was consistent with the finding of Obajimi et al who found lymphadenopathy in $2.0 \%$ of the patient studied [2]. Higher percentages of $37.1 \%, 31.0 \%$ and $17.2 \%$ were recorded by Igbinedion et al., Tshibwabwa et al. and Nzi et al. respectively [3] [24] [29]. Lymph nodes are expected to be more active in the disease due to the increase presence of infections and tumours in these patients. Enlarged abdominal lymph nodes could involve any lymph node group and may be multiple. In this study, most of the lymph node enlargement involved the para aortic and porta hepatis lymph node groups. Majority (56.7\%) of the lymph nodes were seen in patients with $\mathrm{CD}_{4}^{+}$count $\geq 350 / \mu \mathrm{l}$ and $40.0 \%$ in patients with $\mathrm{CD}_{4}^{+}$count $<200 / \mu \mathrm{l}$.

The kidneys can be affected directly by the HIV virus, secondary infections or as a side effect of the drugs used in the HAART. In this study, $6.4 \%$ had increased renal parenchymal echogenicity and $2.4 \%$ had enlarged kidneys. These findings did not correlate with $\mathrm{CD}_{4}^{+}$count $(\mathrm{p}=0.452$ and 0.203 respectively, Table 10). Increased renal parenchymal echogenicity was seen more in patients with $\mathrm{CD}_{4}^{+}$count $\geq 500 / \mu \mathrm{l}$. Similar findings were documented by Adeyekun et al. who found enlarged kidneys in $8.0 \%$ and increased renal echogenicity in $41.7 \%$ of the study population and demonstrated no correlation between renal size and renal parenchymal echogenicity and $\mathrm{CD}_{4}^{+}$count [31]. However Igbinedion et al. recorded a significant correlation between increased renal echogenicity and $\mathrm{CD}_{4}^{+}$count [3]. Renal cyst and calyceal dilatation were seen in $1.2 \%$ and $0.6 \%$ respectively which are non specific and were not investigated further.

Other findings in this study includes ascites which was recorded in $2.4 \%$ with significant correlation with $\mathrm{CD}_{4}^{+}$count $(\mathrm{p}=0.016$, Table 10). Other studies recorded ascites in $1.3 \%$ and $5.7 \%$ respectively which did not correlate with $\mathrm{CD}_{4}^{+}$count [2] [3]. Pelvic abscess was noted in only 1 patient whose $\mathrm{CD}_{4}^{+}$count

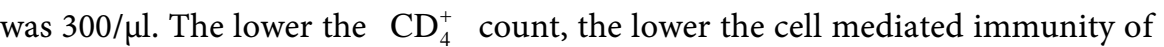
the patient and greater the risk of infections [1]. No patient presented with gastrointestinal findings such as bowel wall thickening in this study. Igbinedion et al. recorded thickened bowel wall in $4.0 \%$ of the study population which correlated significantly with the patients' $\mathrm{CD}_{4}^{+}$count [3].

\section{Conclusion}

The study demonstrated a wide range of abdominal sonographic abnormalities in HIV/AIDS patients. Abdominal lymph node enlargement and ascites correlated significantly with $\mathrm{CD}_{4}^{+}$count. Most of the findings in individual organs did not correlate with $\mathrm{CD}_{4}^{+}$count. This may be related to the fact that most of the patients were in the "Not significant" category $\left(\mathrm{CD}_{4}^{+} \geq 500 / \mu \mathrm{l}\right)$. Although abdominal ultrasound examination is invaluable in the management of these patients, it has not proved to be useful in predicting the patients' immune status.

\section{References}

[1] Sebastian, L. (2002) The Pathology of HIV Infection. Leprosy Review, 73, 64-71.

[2] Obajimi, M.O., Atalabi, M.O., Adeniyi-Sofoluwe, A.T., Adekanmi, A.J. and Osuag- 
wu. Y.U. (2008) Abdominal Ultrasonography in HIV/AIDS Patients in Southwestern Nigeria. BMC Medical Imaging, 8, 5-10. https://doi.org/10.1186/1471-2342-8-5

[3] Igbinedion, B.O., Marchie, T.T. and Ogbeide, E. (2009) Trans-Abdominal Ultrasound Findings Correlated with $\mathrm{CD}_{4}^{+}$Counts in Adults HIV-Infected Patients in Benin Nigeria. SA Journal of Radiology, 13, 34-40.

[4] Gore, R.M., Miller, F.H. and Yaghmai, V. (1998) Acquired Immune Deficiency Syndrome of the Abdominal Organs: Imaging Features. Seminars in Ultrasound, $C T$, and $M R, 19,175-189$. https://doi.org/10.1016/S0887-2171(98)90059-2

[5] Goldin, R.D. and Lloyd, J. (2002) HIV and Hepatobiliary Disease. Current Diagnostic Pathology, 8, 144-151. https://doi.org/10.1054/cdip.2002.0112

[6] Flum, D.R., Steinberg, S.D., Sarkis, A.Y., Pacholka, J.R. and Wallack, M.K. (1997) The Role Of Cholecystectomy in Acquired Immunodeficiency Syndrome. Journal of the American College of Surgeons, 184, 233-239.

[7] Guarda, L.A., Stein, S.A., Cleary, K.A. and Ordonez, N.G. (1983) Human Cryptosporidiosis in the Acquired Immune Deficiency Syndrome. Archives of Pathology \& Laboratory Medicine, 107, 562-566.

[8] Pitlik, S.D., Fainstein, V., Rios, A., Guarda, L. and Mansell, P.W. (1983) Cryptosporidial Cholecystitis. New England Journal of Medicine, 308, 127-129.

[9] Ko, W.F., Cello, J.P., Rogers, S.J. and Lecours, A. (2003) Prognostic Factors for the Survival of Patients with AIDS Cholangiopathy. American Journal of Gastroenterology, 98, 2176-2181. https://doi.org/10.1111/j.1572-0241.2003.07718.x

[10] Enns, R. (2003) AIDS Cholangiopathy: “An Endangered Disease”. American Journal of Gastroenterology, 98, 2111-2112.

[11] O’Hara, S.P., Small, A.J., Gajdos, G.B., Badley, A.D., Chen, X.M. and Laruss, N.F. (2009) HIV-1 Tat Protein Suppresses Cholangiocyte Toll-Like Receptor 4 Expression and Defense against Cryptosporidium parvum. The Journal of Infectious Diseases, 199, 1195-1204. https://doi.org/10.1086/597387

[12] Mahajani, R.V. and Uzer, M.F. (1999) Cholangiopathy in HIV-Infected Patients. Clinical Liver Disease, 3, 669-684.

[13] Bouche, H., Housset, C. and Dumont, J.L. (1993) AIDS-Related Cholangitis: Diagnostic Features and Course in 15 Patients. Journal of Hepatology, 17, 34-39.

[14] Cello, J.P. and Chan, M.F. (1995) Long-Term Follow-Up of Endoscopic Retrograde Cholangiopancreatography Sphincterotomy for Patients with Acquired Immune Deficiency Syndrome Papillary Stenosis. American Journal of Medicine, 99, 600-603.

[15] Cello, J.P. (1989) Acquired Immunodeficiency Syndrome Cholangiopathy: Spectrum of Disease. American Journal of Medicine, 86, 539-546.

[16] Cheter, E.Z., Lango, M.A. and Laudanna, A.A. (2000) Involvement of the Pancreas in AIDS: A Prospective Study of 109 Post-Mortems. AIDS, 14, 1879-1886. https://doi.org/10.1097/00002030-200009080-00001

[17] Wyatt, C.M., Morgello, S. and Katz-Malamed, R. (2009) The Spectrum of Kidney Disease in Patients with AIDS in the Era of Antiretroviral Therapy. Kidney International, 75, 428-434. https://doi.org/10.1038/ki.2008.604

[18] Kalim, S., Szczech, L.A. and Wyatt, C.M. (2008) Acute Kidney Injury in HIV-Infected Patients. Seminars in Nephrology, 28, 556-562.

[19] Atta, M.G., Deray, G. and Lucas, G.M. (2008) Antiretroviral Nephrotoxicities. Seminars in Nephrology, 28, 563-575.

[20] U.S. Department of Health and Human Services (2011) Health Resources and Services Administration (HRSA), HIV/AIDS Bureau. Guide for HIV/AIDS Clinical 
Care.

[21] Cassani, F., Valentini, P., Cataleta, M., Manotti, P. and Francesconi, R. (1997) Ultrasound-Detected Abdominal Lymphadenopathy in Chronic Hepatitis C: High Frequency and Relationship with Viremia. Journal of Hepatology, 26, 479-483.

[22] World Health Organization (2006) WHO Case Definitions of HIV for Surveillance and Revised Clinical Staging and Immunological Classification of HIV-Related Disease in Adults and Children. World Health Organization, Geneva, 16.

[23] Kawooya, M.G. (2013) Abdominal Ultrasound Findings in HIV and Tuberculosis. Imaging in Medicine, 5, 265-274. https://doi.org/10.2217/iim.13.22

[24] Tshibwabwa, E.T., Mwaba, P., Bogle-Taylor, J. and Zumla, A. (2000) Four-Year Study of Abdominal Ultrasound in 900 Central African Adults with AIDS Referred for Diagnostic Imaging. Abdominal Imaging, 25, 290-296. https://doi.org/10.1007/s002610000035

[25] Adeoye, S. (2005) Sexual Behavior, Perception of HIV/AIDS and Condom Use among Commercial Motorcyclists in Benin City. Nigerian Postgraduate Medical Journal, 12, 262-265.

[26] Federal Ministry of Health (2005) National HIV/Syphilis Sero-Prevalence Sentinel Survey.

[27] Smith, F.L., Matheson, J.R. and Coopersberg, P.L. (1994) Abdominal Abnormalities in AIDS. Detection at Ultrasound in a Large Population. Radiology, 192, 691-695. https://doi.org/10.1148/radiology.192.3.8058935

[28] Pawar, S.S., Biradar, S.P., Gajbhiye, M.I. and Khadse, G.J. (2013) Correlation of Hepatobiliary Ultrasound Findings with CD4+ Count and Liver Function Tests in HIV Positive Patients. Medical Journal of Western India, 41, 86-88.

[29] N’Zi, P.K., Coulibaly, A., N’Dri, K., Quattara, N.D. and Diabate, S.A. (1999) Ultrasound Aspect Abdominal Involvement in Adults with HIV Infections in the Ivory Coast: A Propos of 146 Cases. Sante, 9, 85-88.

[30] Sawhney, M.P.S. (2008) Lymphoreticular Involvement in Human Immunodeficiency Virus Infection. $M J A F I, 64,4-6$.

[31] Adeyekun, A.A., Unuigbe, E.I., Onunu, A.N. and Azubike, C.O. (2011) Renal Sonographic Parameters in Human Immunodeficiency Virus-Infected Subjects and Relationship to CD4 Cell Count. Saudi Journal of Kidney Diseases and Transplantation, 22, 1164-1168.

\section{Scientific Research Publishing}

Submit or recommend next manuscript to SCIRP and we will provide best service for you:

Accepting pre-submission inquiries through Email, Facebook, LinkedIn, Twitter, etc. A wide selection of journals (inclusive of 9 subjects, more than 200 journals)

Providing 24-hour high-quality service

User-friendly online submission system

Fair and swift peer-review system

Efficient typesetting and proofreading procedure

Display of the result of downloads and visits, as well as the number of cited articles

Maximum dissemination of your research work

Submit your manuscript at: http://papersubmission.scirp.org/

Or contact ami@scirp.org 